\title{
Abstracts from Nippon Eiseigaku Zasshi (Japanese Journal of Hygiene) vol. 69, no.1
}

Published online: 20 March 2014

(C) The Japanese Society for Hygiene 2014

\section{Clarification of Molecular Targets of Dioxin Toxicity}

Nippon Eiseigaku Zasshi, 69, 1-7 (2014)

Chiharu Tohyama

Laboratory of Environmental Health Sciences CDBIM, Graduate

School of Medicine, The University of Tokyo

Health effects associated with exposure to various chemicals have been extensively studied. However, in most cases, the molecular basis of the underlying mechanism has been elusive. Dioxin toxicity, which has raised a significant concern in society, was discovered to be mediated by a high-affinity receptor, aryl hydrocarbon receptor (AhR), more than three decades ago. This receptor has been established to be essential for the manifestation of various toxicities, such as carcinogenicity, reproductive toxicity, developmental neurotoxicity, and immunotoxicity. However, it has not been clarified how AhR mediates such a wide variety of dioxin toxicities through AhR-dependent mechanisms. In recent years, several lines of experimental evidence have provided clues for opening the "black box" that contains the molecular mechanisms of dioxin action. In this review, I focus on dioxin toxicity phenotypes for which downstream molecular targets have begun to be elucidated. The toxicity phenotypes include impaired prostate development and hydronephrosis in mouse fetuses and pups, respectively, as well as abnormality in organogenesis in zebrafish embryos and adults. As the molecular basis of the tissue-specific endpoints of dioxin toxicity, dysregulation of AhR downstream pathways, such as signaling of prostanoid synthesis, Wnt/ $\beta$-catenin signaling, and signaling by receptors for inflammatory cytokines, are discussed. The new findings on the molecular targets of dioxin may provide clues to the prospective discovery of new molecular events associated with the growth and development of organs and pathogenesis of diseases.

\section{Application of Molecular-targeting Cancer Prevention to Tumor Immunity}

Nippon Eiseigaku Zasshi, 69, 8-14 (2014)

Mano Horinaka

Department of Molecular-Targeting Cancer Prevention, Graduate

School of Medical Science, Kyoto Prefectural University of Medicine

The development of methods of cancer prevention is a particularly important issue. We have proposed a strategy termed "combination- oriented molecular-targeting prevention" of cancer. As the molecular target of our model, we focused on the tumor necrosis factor-related apoptosis-inducing ligand (TRAIL) and its receptor. TRAIL is a cytokine from immune cells and is important for immune surveillance and defense mechanisms against cancer cells. First, we searched for food factors inducing the expression of death receptor 5 (DR5), one of the TRAIL receptors. DR5 is specifically expressed on the membrane of cancer cells, not on that of normal cells. We found that many food factors markedly induced DR5 expression, enhancing TRAIL sensitivity against cancer cells. We next found that lactic acid bacteria induced endogenous TRAIL production in human peripheral blood mononuclear cells (PBMCs). Importantly, lactic acid bacteria increased the NK activity of PBMCs against cancer cells. Moreover, we found that butyric acid bacterium, one of the antiflatulents, induced the release of endogenous TRAIL from polymorphonuclear neutrophils. The butyric acid bacterium was proven effective against cancer cells by inducing apoptosis in vivo as well as in vitro. Taken together, these results strongly suggest that the strategy of "combination of DR5inducing food factors and TRAIL-inducing food factors" as moleculartargeting prevention of cancer will be a practical approach.

\section{Role of Zinc in Type 2 Diabetes}

Nippon Eiseigaku Zasshi, 69, 15-23 (2014)

Motoyuki Tamaki, Yoshio Fujitani

Department of Metabolism and Endocrinology, Juntendo University Graduate School of Medicine

Pancreatic $\beta$ cells contain the highest amount of zinc among cells within the human body, and hence, the relationship between zinc and diabetes has been of great interest. To date, many studies of zinc and diabetes have been reported, including studies demonstrating that diabetic patients and mice have a decreased amount of zinc in the pancreas. Zinc may counteract the deleterious effects of oxidative stress, which contributes to reduced insulin resistance, and may also protect pancreatic $\beta$ cells from glucolipotoxicity. Recently, we have shown that SLC30A8/zinc transporter 8, which is a transporter expressed on the surface of insulin granules, plays a key role in zinc transport into insulin granules and in the regulation of hepatic insulin clearance. Here, we review the role of zinc in whole-body maintenance and the latest information on the relationship between zinc and diabetes. 


\section{Psychophysiological Effects of Hand Massage in Geriatric Facility Residents}

Nippon Eiseigaku Zasshi, 69, 24-30 (2014)

Namiko Ogawa, ${ }^{*}$, Kumiko Kuroda ${ }^{*, 1}$, Satoshi Ogawara, ${ }^{*}$, Naoyuki Miyake, ${ }^{*, 2}$, Kazuhiko Machida ${ }^{*}, 1$

${ }^{*}$, Graduate School of Human Sciences, Waseda University,

${ }^{*}, 2$ Geriatric Health Services Facility "SANTOME"

Objectives: Geriatric facility residents experience easy decrease of their quality of life (QOL) compared with elderly people cared for in their own homes owing to environmental changes. Because complementary and alternative medicine is useful to improve QOL, a simple manual for hand massage for inexperienced volunteers was developed, and the physical and psychological effects of this method were examined.

Methods: Thirty-six female geriatric facility residents (mean age 84.5 years) were chosen. They were divided into two groups: the control and hand-massaged groups. In the hand-massaged group, females received $15 \mathrm{~min}$ hand massage from volunteers in accordance with a simple manual. No hand massage was performed in the control group. Before and after the treatment, a survey using a questionnaire (7 items; e.g., tension, fatigue) and physiological measurements (e.g., blood pressure, hand skin temperature), and measurement of cortisol and chromogranin A levels in saliva samples using enzyme-linked immunosorbent assay kits were carried out. Data were analyzed by twoway analysis of variance (ANOVA) to determine the effects of group and time on the dependent variables.

Results: The hand-massaged group showed greater increases in skin temperature than the control group, as shown by ANOVA $(p<.05)$. Results showed that improvements of the scores in the questionnaire (condition and feel-good items) were greater in the hand-massaged group than in the control group $(\mathrm{p}<.05)$. Cortisol level increased in the control group, but was not statistically significantly different from that in the hand-massaged group, as shown by ANOVA. Chromogranin A level was also not significantly different between the two groups.

Conclusions: These results confirmed the benefits of complementary and alternative medicine including touching and massage found in previous studies. Hand massage prompted relaxation in geriatric facility residents in this experiment.

\section{Measurement of Hazardous Chemical Constituents and Mutagenic Activity in Fillers and Mainstream Smoke from Neo Cedar}

Nippon Eiseigaku Zasshi, 69, 31-38 (2014)

Yohei Inaba ${ }^{*, 1}$, Tadamichi Ohkubo ${ }^{*}, 1$, Kazutoshi Sugita ${ }^{*}, 2$, Shigehisa Uchiyama ${ }^{*}, 1$, Hiromitsu Ogata ${ }^{*, 3}$, Naoki Kunugita ${ }^{*, 1}$

${ }^{*}, 1$ Department of Environmental Health, National Institute of Public Health, ${ }^{*}, 2$ Mitsubishi Chemical Analytech Co., Ltd., ${ }^{*}, 3$ Center for Public Health Informatics, National Institute of Public Health

Objective: To determine constituents of fillers and mainstream smoke from Neo Cedar.

Methods: Neo Cedar is a second-class over-the-counter (OTC) drug and similar to cigarettes in a number of ways. In particular, the design and usage are very similar to those of cigarettes. For the fillers of the drug, the levels of nicotine, tobacco-specific nitrosamines (TSNA), and heavy metals, and mutagenicity were determined using the methods for cigarette products. For the mainstream smoke, the levels of tar, nicotine, carbon monoxide (CO), TSNA, polycyclic aromatic hydrocarbons (PAH), and carbonyl compounds were also determined using the methods for cigarettes. The mainstream smoke from the drug was collected with a smoking machine using two smoking protocols (ISO and Health Canada Intense methods).

Results: The nicotine and total TSNA levels in the fillers of the drug averaged $2.86 \mathrm{mg} / \mathrm{g}$ and $185 \mathrm{ng} / \mathrm{g}$, respectively. The nine species of heavy metals were also detected in the fillers of the drug. The levels of nicotine, tar, CO, TSNA, PAH, and carbonyl compounds of mainstream smoke from the drug were higher when determined using the HCI regime than when using the ISO regime. The mutagenicity of the mainstream smoke determined using the HCI regime was also higher than that determined using the ISO regime.

Conclusion: In this study, all constituents of Neo Cedar were determined by methods for cigarette products. The drug had a ventilation hole on its filter. Thus, its constituents are different from those determined by the smoking protocols. Neo Cedar users should be careful of higher exposure to the hazardous gases owing to smoking patterns.

\section{Effect of Variation of Lemon Intake and Walking in Daily Life on Various Indicators of Muscle Mass and Blood Biochemistry in Menopausal Middle-aged and Elderly Women}

Nippon Eiseigaku Zasshi, 69, 39-48 (2014)

Kimiko Sato ${ }^{*}, 1$, Tokio Domoto ${ }^{*}, 1$, Masanori Hiramitsu ${ }^{*, 2}$, Takao Katagiri $^{*}, 2$, Yoji Kato ${ }^{*}, 1$, Yukiko Miyake ${ }^{*}, 1$, Katsuhide

Ishihara $^{*}, 1$, Namiko UMEI ${ }^{*}, 1$, Atsushi Takigawa ${ }^{*}, 1$, Toshihide Harada $^{*, 1}$, Satomi Aoi ${ }^{*}, 1$, Hiromi Ikeda ${ }^{*}, 1$

${ }^{*}, 1$ Prefectural University of Hiroshima, ${ }^{* 2}$ Incorporated Company POKKA CORPORATION Central Research Laboratory

Objectives: We examined the factors considered to change body composition and blood biochemistry indicators in menopausal middle-aged and elderly women. These changes result from exercise by walking as part of their daily activities and lemon consumption by women who live on the small islands of the Seto Inland Sea, Japan's largest citrus fruit (lemon)-producing region.

Methods: Between September 2011 and March 2012, we recorded the daily lemon consumption and the number of steps taken by 101 middle-aged and elderly female lemon farmers. We also measured their body dimensions, body compositions, and blood pressure pulse wave velocity and conducted blood tests before and after the survey period. The results before and after the survey period were compared by the $t$ test and associations were determined on the basis of Pearson's correlation coefficient. Covariance structural analysis was carried out to determine causal associations.

Results: From the results of covariance structure analysis, lemon intake did not have a direct impact on each item examined. The third item, i.e., "the factors related to arteriosclerosis," was affected indirectly via citric acid and fatigue, and anticoagulation was shown. The fourth item, i.e., "the factors related to maintenance of muscle mass," which is affected by menopausal years and the change in walking speed, was shown to be associated with the second item, i.e., "the factors related to lipid metabolism." Menopausal years affected the first, third and fourth items.

Conclusions: Lemon intake did not have a direct impact on each item. Lemon has been shown to indirectly affect the third item through citric acid. Walking affected the second item, the level of total cholesterol, such as HDL cholesterol, through the fourth item. The importance of providing services that lead to sustained physical activity and a well-balanced metabolism between lipids and carbohydrates has been shown. 


\section{Challenges and Development of a Nutritional Management Service System Home Page that Targets Personal Computer Novices 65 Years of Age or Older}

Nippon Eiseigaku Zasshi, 69, 49-59 (2014)

Kimiko Sato, Atsushi Hosokawa, Seiji Nishida, Akemi Kariya, Satoshi Imaizumi

Prefectural University of Hiroshima

Objectives: We intended to develop contents for nutritional management for elderly people using the Internet, and to consider factors related to the promotion of Information and Communication Technology (ICT) use.

Methods: A questionnaire survey was carried out consisting of items on diet support conditions and promoting the use of ICT by the elderly. Then, we developed a nutritional management system using a home page on a trial basis, after which $\mathrm{n}$ we studied the need, operability and environmental support of this system.

Results: Regarding the state of ICT use more than one- half of the respondents were daily users of the Internet $62.4 \%$, and mobile phones $65.2 \%$. On the four items on diet, such as "buy the same food, forget to buy food", 22.7 \%responded that "forget to buy food", which was the highest, and interest in "lunch home delivery" showed a low percentage. Among the respondents $19.6 \%$ answer "yes" to the question that memory, "have trouble remembering where you put things" and $13.4 \%$ responded that they forget to take medicine". There was a positive correlation of what with five items on operability, "What is simple and easy to use is good" and the effectiveness of personal computers and mobile phones has been shown. In addition, $32.3 \%$ responded "When the operation is difficult, the use becomes inconvenient".

Conclusions: It was suggested that by setting up an opportunity to experience the effectiveness and the satisfaction of using a system that addresses elderly people's concerns, we can modify attitudes and provide motivation, reducing the sense of weakness or irrelevance that can hinder the use of a nutritional management system. It was suggested that along with the sense of effectiveness of screen displays with color instructions and simple design that match the physical characteristics of the elderly, the sense of being able to operate the system afterwards will affect the need for ICT use and utilization rate.

\section{Present Status of Expanded Newborn Screening Project for Inborn Errors of Metabolism by Tandem Mass Spectrometry}

Nippon Eiseigaku Zasshi, 69, 60-74 (2014)

Tomiko Kuhara

Japan Clinical Metabolomics Institute

In Japan, screening for six diseases including four inborn errors of metabolism has been performed since 1977 for all neonates to prevent severe mental handicaps or death. A rapid screening procedure for analysis of several amino acids and acylcarnitines in blood spots by tandem mass spectrometry was developed by Millington DS et al. in the early 1990s. Although it is called expanded (or extended) newborn screening, the procedure is insufficiently sensitive to or specific for several diseases. Screening for all diseases that can be screened using this procedure is suggested to be cost-ineffective. Many European countries target only two diseases: medium-chain acyl-CoA dehydrogenase deficiency and phenylketonuria; their prevalence in Caucasian populations is very high, but some countries target more than twenty diseases and others an intermediate number. A pilot study targeting 22 diseases suggests that the combined incidence is one per $9,000(0.01 \%)$ in Japan. This primary screening requires secondary screening to confirm the disease using urine, and either organic acids with solvent extraction or metabolome without fractionation are analyzed by gas chromatography-mass spectrometry. There is no need for primary or secondary screening tests to be performed at the same laboratory because the skills required are quite different. Understanding of the methodological problems of tandem mass screening and amelioration of variation and false positivity rate of this screening method among laboratories are critical to the success of the screening system in Japan. GC/MS-based urine metabolomics is expected to become one of the primary screening methodologies for neonates/ infants who are already ill.

\section{History of Ignorance of Methylmercury Toxicity and Intoxication in Japan in Relation to Minamata Disease}

Nippon Eiseigaku Zasshi, 69, 75-79 (2014)

Nobuo Ishihara

Kanagawa Health Service Association

The first reports of methylmercury intoxication appeared in 1865 and 1866. These reports had sensational effects in European countries, and were introduced not only in journals but also in newspapers. These two reports were referable in Japan at the latest in 1927. The formation of organic mercury in the production of acetoaldehyde was also referable in 1906 in Japan. In 1931 (1 year before the start of acetoaldehyde production in Minamata) these important reports cited above were referable in Kumamoto University, and there were warnings about the toxicity of organic mercury and environmental pollution prior to the start of acetoaldehyde production. However, not only the plant, authorities (Ministry of Welfare), and Kumamoto Prefectural Office, but also the scientists completely ignored these reports. Waste was dumped into the environment without any treatment. Serious pollution of the environment by organic mercury started, which resulted in the outbreak of Minamata disease (=methylmercury intoxication). 